Article

\title{
Analysis of Subsynchronous Resonance Characteristics and Influence Factors in a Series Compensated Transmission System
}

\author{
Chengbing $\mathrm{He}^{1, *}$, Dakang Sun ${ }^{2}$, Lei Song ${ }^{3}$ and Li Ma ${ }^{1}$ \\ 1 School of Energy, Power and Mechanical Engineering, North China Electric Power University, \\ Beijing 102206, China \\ 2 Information Technology Department, Guodian Nanjing Automation Corporation, Nanjing 210000, China \\ 3 Technology and Engineering Center for Space Utilization, Chinese Academy of Sciences, \\ Beijing 102206, China \\ * Correspondence: hcbyy@ncepu.edu.cn; Tel.: +86-010-61772359
}

Received: 4 July 2019; Accepted: 21 August 2019; Published: 26 August 2019

\begin{abstract}
Series capacitor compensation is used to improve the utilization of existing power systems. Subsynchronous resonance (SSR) can be caused by series compensated lines, which would lead to turbogenerator shaft breakdown. A novel approach was presented in this paper to analyze the characteristics and influence factors of SSR in a series compensated transmission system. The system model of SSR, including the various modules of the electromechanical network, was established, and the eigenvalue results under $70 \%$ series compensation level were analyzed by eigenvalue analysis method for the the institute of electrical and electronics engineers (IEEE) first benchmark model (FBM). Compared with the results of the power systems computer-aided design (PSCAD) modeling and simulation, the effectiveness of eigenvalue analysis method was proven. After that, the eigenvalue analysis method was used to study, in detail, the effects of system series compensation levels, synchronous generator parameters, speed governing system parameters, and excitation system parameters on SSR characteristics. The research results show that the series compensation level has the greatest influence on the torsional mode damping of the system. The parameters of generator reactance, speed governing system, and excitation system have some effect on the torsional mode damping. The parameters of excitation system significantly affect the low-frequency oscillation damping.
\end{abstract}

Keywords: eigenvalue analysis method; series compensated transmission; subsynchronous resonance (SSR); torsional mode (TM)

\section{Introduction}

Series capacitor compensation used in long-distance transmission systems can enhance the transfer capability and transient stability. However, it is well-known that capacitors in series with transmission lines can cause subsynchronous resonance (SSR) in power systems. Self-excitation or dynamic instability due to SSR produces very high amplitude mechanical torques in the turbogenerator shaft that could lead to shaft damage [1,2]. The first reported case of SSR in the world occurred at Mohave Power Station in Arizona, where the generator shafting was subjected to a continuously increasing torque, which led to the shafting fracture. After that, several cases of shaft damage caused by SSR have happened. For example: In 2004, the generator rotors of 2\# and 3\# turbogenerators set cracked due to SSR in Dresden nuclear power plant. In 2008, several SSR events occurred at Yimin power plant in China, which led to the shaft fracture of $3 \#$ turbogenerator set. In recent years, wind power has been developing rapidly worldwide, and there have been many incidents of damage to the wind 
farm equipment caused by SSR [3-7]. For example: In 2009, SSR occurred at the Zorillo-Gulf wind farms. This incident caused multiple transmission devices to trip and many crowbar circuits to fail. During the year 2013, dozens of SSRs occurred in the Guyuan wind power system in Hebei Province, China. More than one thousand wind turbine generators tripped due to overcurrent caused by SSR, and the power of the wind farm dropped sharply in a short time. SSR seriously affects the safe and reliable operation of power systems, which has attracted great attention of countries all over the world.

There are many methods to study SSR in the power system. Amongst these, the eigenvalue analysis, electromagnetic transient analysis, complex torque coefficient analysis, and frequency scanning are the most used methods [8-11]. A summary of several major analysis methods is shown in Table 1.

Table 1. Comparison of subsynchronous resonance (SSR) analysis methods.

\begin{tabular}{|c|c|c|c|}
\hline SSR Analysis Methods & Features & Advantages & Disadvantages \\
\hline Eigenvalue analysis & $\begin{array}{l}\text { It is also called state-space } \\
\text { analysis method, which is } \\
\text { a quantitative analysis method } \\
\text { based on the small disturbance } \\
\text { linearization model and obtains } \\
\text { SSR-related information by } \\
\text { solving eigenvalues. }\end{array}$ & $\begin{array}{l}\text { Provides frequency and } \\
\text { damping information for } \\
\text { entire system in } \\
\text { one calculation. } \\
\text { - Analyzes the influence of } \\
\text { parameters on SSR system. }\end{array}$ & $\begin{array}{l}\text { Higher order model } \\
\text { parameters are required for } \\
\text { the entire system, which is } \\
\text { difficult to analyze. }\end{array}$ \\
\hline $\begin{array}{l}\text { Electromagnetic } \\
\text { transient analysis }\end{array}$ & $\begin{array}{l}\text { Using a step-by-step numerical } \\
\text { integration method, a set of } \\
\text { differential equations of the } \\
\text { system are solved. } \\
\text { The mathematical model can be } \\
\text { linear or nonlinear. }\end{array}$ & $\begin{array}{l}\text { The dynamical } \\
\text { characteristics of all power } \\
\text { system components can } \\
\text { be represented. } \\
\text { - Has a unified simulation } \\
\text { software tools such as } \\
\text { EMTP, PSCAD, EMTDC. }\end{array}$ & $\begin{array}{l}\text { Only time domain response } \\
\text { results can be given. It is } \\
\text { difficult to directly give SSR } \\
\text { reasons and the mechanism } \\
\text { of instability. }\end{array}$ \\
\hline $\begin{array}{l}\text { Complex torque } \\
\text { coefficient analysis }\end{array}$ & $\begin{array}{l}\text { Frequency scanning of the } \\
\text { mechanical and electrical } \\
\text { complex torque coefficients of } \\
\text { the shaft system in the } \\
\text { subsynchronous frequency } \\
\text { range to determine whether the } \\
\text { system will undergo } \\
\text { subsynchronous oscillation. }\end{array}$ & $\begin{array}{l}\text { Damping frequency curve } \\
\text { can be obtained. } \\
\text { The influence of various } \\
\text { parameters on the electrical } \\
\text { damping characteristics } \\
\text { can be analyzed. }\end{array}$ & $\begin{array}{l}\text { - Large time-frequency } \\
\text { analysis error. } \\
\text { Only applicable to } \\
\text { single machine infinite } \\
\text { bus system. }\end{array}$ \\
\hline Frequency scanning & $\begin{array}{l}\text { It is an approximate linear } \\
\text { method to calculate the } \\
\text { equivalent impedance for } \\
\text { a specific frequency and filter } \\
\text { out the system conditions with } \\
\text { potential SSR. }\end{array}$ & $\begin{array}{l}\text { - Simple theory and } \\
\text { less calculation. } \\
\text { - Can quickly determine if } \\
\text { there is a SSR risk. }\end{array}$ & $\begin{array}{l}\text { Only electrical } \\
\text { resonance points can } \\
\text { be obtained. } \\
\text { Can only be judged } \\
\text { qualitatively and with } \\
\text { poor accuracy. }\end{array}$ \\
\hline
\end{tabular}

The purpose of this paper was to study the SSR characteristics and influence factors of a series compensated transmission system. It can be seen from Table 1 that the combination of eigenvalue analysis method and time domain simulation method can well meet the requirements of accurate analysis. SSR system model, including a variety of electromechanical network modules, is the established. The eigenvalues under $70 \%$ series compensation level are analyzed by eigenvalue analysis method for the institute of electrical and electronics engineers (IEEE) first benchmark model (FBM). At the same time, power systems computer-aided design (PSCAD) is used to model and analyze the example, which proves the validity of eigenvalue analysis method. After that, the effects of series compensation levels, synchronous generator parameters, speed governing system parameters, and excitation system parameters on SSR characteristics of the system are studied in detail. 


\section{Eigenvalue Analysis and System Modeling}

\subsection{Eigenvalue Analysis}

Eigenvalue analysis is a strict and accurate method based on linear theory. In order to analyze SSR characteristics by eigenvalue analysis method, it is necessary to establish the detailed mathematical model of each system component. Then, the linear state-space model is obtained by linearizing the system at the stable operating points.

$$
\dot{X}=A X
$$

where $X$ is the system state variables of the incremental form, and $A$ is the state matrix of the system. The corresponding eigenvalue equation of the system is [8]

$$
|\lambda I-A|=0
$$

where $I$ is identity matrix. $\lambda$ is the eigenvalues of matrix $A$, and it can be expressed as

$$
\lambda_{i}=\sigma_{i} \pm j \omega_{i}
$$

In summary, the steps of eigenvalue analysis are as follows:

- A detailed mathematical model is established for various components of the SSR system and the power network, and the differential equations describing the transient process is obtained.

- By linearizing the differential equations near stable values and writing out the standard form $\dot{X}=A X$, the state-space representation is acquired, which is suitable for eigenvalue analysis.

- The initial values of the studied system under stable operation are calculated.

- The eigenvalues of characteristic equations are solved, and the stability and damping characteristics of the system are analyzed based on the results.

\subsection{System Modeling}

The IEEE FBM, which was created by the IEEE Working Group in 1977, is used for SSR studies [12]. The system is shown in Figure 1.

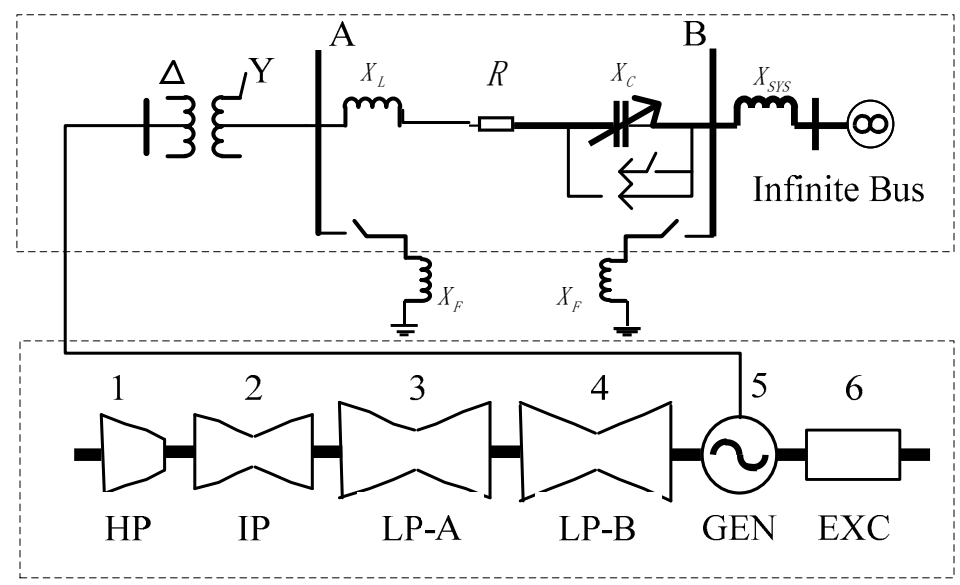

Figure 1. The IEEE first benchmark model [12].

The system consists of a turbogenerator shaft system, which includes high pressure turbine (HP), intermediate pressure turbine (IP), low pressure turbine A (LP-A), low pressure turbine B (LP-B), generator (GEN), and exciter (EXC), and an infinite bus with a series compensated transmission line. The system models include turbogenerator shaft system, turbine speed governing system, turbine prime mover system, generator electromagnetic circuits, exciter system, transmission lines, 
and series compensation circuit system. The models of the turbine speed governing system and turbine prime mover system are as follows. Other models are given in [13].

\subsubsection{Turbine Speed Governing System}

The main function of the speed governing system of a modern large turbogenerator is to adjust the speed and load of the system. The state characteristic equations of the speed governing system at an operating point are established as follows [8].

$$
\left\{\begin{array}{l}
\mathrm{p} \Delta \alpha=-\frac{K_{G}}{T_{S R}} \Delta \omega-\frac{1}{T_{S R}} \Delta \alpha \\
p \Delta \mu=\frac{1}{T_{S M}} \Delta \alpha-\frac{1}{T_{S M}} \Delta \mu
\end{array}\right.
$$

where $T_{S M}$ is the integral time constant of the oil motive, $K_{G}$ and $T_{S R}$ are the amplification factor and time constant of the speed regulator, respectively. $\alpha$ and $\mu$ are the position of the speed relay and the opening of the turbine inlet valve, respectively.

\subsubsection{Turbine Prime Mover Model}

Figure 2 is a typical turbine prime mover system model. Where $P_{G V}$ is the valve opening signal output from electro-hydraulic servomechanism of the speed governing system. $T_{\text {css }}$ is the steam volume time constant of the high pressure turbine. $T_{\text {rhs }}$ is the steam volume time constant of the reheater. $T_{\cos }$ is the steam volume time constant of the connected pipe. FHP, FIP and FLP are the power ratios of high pressure turbine, medium pressure turbine, and low pressure turbine, respectively.

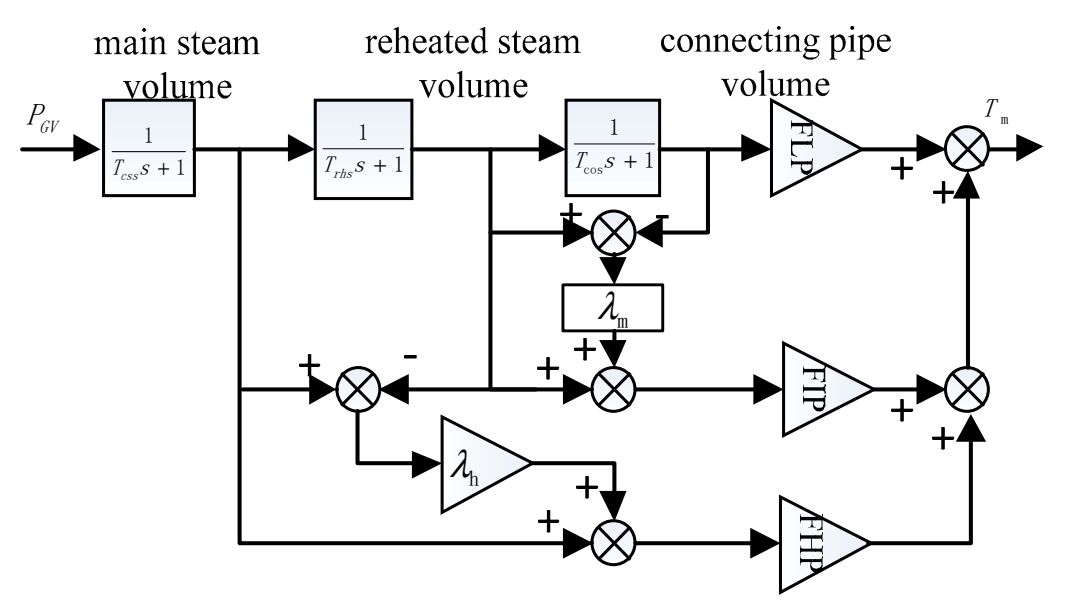

Figure 2. Principle block diagram of turbine prime mover transfer function [9].

According to mathematical models of turbine prime mover, the state-space model can be established, and the state characteristic equations at the operating point can be obtained as follows [9]:

$$
\left\{\begin{array}{l}
P \Delta T_{1}=-\frac{1}{T_{C S S}} \Delta T_{1}+\frac{F H P+F H P \cdot \lambda_{\mathrm{h}}}{T_{C S S}} \Delta P_{G V} \\
P \Delta T_{2}=-\frac{1}{T_{r h s}} \Delta T_{2}+\frac{1}{T_{r h s}} \cdot \frac{F P+F I P \cdot \lambda_{\mathrm{m}}-F H \cdot \lambda_{\mathrm{h}}}{F H P P+F P P \cdot \lambda_{\mathrm{h}}} \Delta T_{1} \\
p \Delta T_{3}=-\frac{1}{T_{\cos }} \Delta T_{3}+\frac{1}{T_{\cos }} \cdot \frac{F L P F I P \cdot \lambda_{\mathrm{m}}}{F I P+F I P \cdot \lambda_{\mathrm{m}}-F H P \cdot \lambda_{\mathrm{h}}} \Delta T_{2} \\
\Delta T_{4}=\Delta T_{3}
\end{array}\right.
$$

where $T_{1}, T_{2}$ and $T_{3}$ are the mechanical torques of the high pressure turbine, the intermediate pressure turbine, and the low pressure turbine, respectively.

All the above equations consist of the system characteristic equations, including 27 first-order linear differential equations and 27 state variables, which includes 12 state variables of turbogenerator shaft, $X_{s}=\left[\Delta \omega_{1}, \Delta \omega_{2}, \Delta \omega_{3}, \Delta \omega_{4}, \Delta \omega_{5}, \Delta \omega_{6}, \Delta \delta_{1}, \Delta \delta_{2}, \Delta \delta_{3}, \Delta \delta_{4}, \Delta \delta_{5}, \Delta \delta_{6}\right]^{T}$, where $\omega_{i}$ is the angular velocity, 
and $\delta_{i}$ is the angular displacement of mass i. Two state variables of turbine speed governing system, $X_{g}=[\Delta \alpha, \Delta \mu]^{T}$; three state variables of turbogenerator prime movers, $X_{y}=\left[\Delta T_{1}, \Delta T_{2}, \Delta T_{3}\right]^{T}$; six state variables of generator electromagnetic voltage circuit, $X_{G}=\left[\Delta i_{d}, \Delta i_{f}, \Delta i_{D}, \Delta i_{q}, \Delta i_{g}\right]^{T}$, where $i_{d}, i_{f}, i_{D}, i_{q}, i_{g}$ and $i_{Q}$ are the currents through six windings of the generator, which are stator equivalent $\mathrm{d}$-axis winding, excitation winding, $\mathrm{d}$-axis damper winding $\mathrm{D}$, stator equivalent $\mathrm{q}$-axis winding, $\mathrm{q}$-axis damping winding $g$ and $Q$, respectively; two state variables of excitation system, $X_{\mathrm{e}}=\left[\Delta u_{R}, \Delta E_{f d}\right]^{T}$, where $u_{R}$ and $E_{f d}$ are an intermediate variable of the excitation system and the output voltage of the exciter, respectively; two state variables of series compensation capacitors, $X_{A}=\left[\Delta u_{c d}, \Delta u_{c q}\right]^{T}$, where $u_{c d}$ and $u_{c q}$ are the components of the voltage across the series compensation capacitor at the $\mathrm{d}$-axis and the q-axis, respectively.

\section{Case Analysis}

\subsection{Model Parameters}

The IEEE FBM model shown in Figure 1 is used for SSR studies. The system specific parameters and initial state variables are as follows [12].

A. Turbogenerator shaft parameters:

The shaft system of the turbogenerator is modeled as a multi-mass system, including four turbine sections, a generator, and an exciter. Turbogenerator shaft parameters are shown in Table 2.

Table 2. Turbogenerator shaft parameters [12].

\begin{tabular}{cccc}
\hline Mass Block & Inertia Constant, $\boldsymbol{T}_{\boldsymbol{J}}(\mathbf{s})$ & Shaft Segment & Elastic Constant, K (p.u.) \\
\hline HP & 0.185794 & - & - \\
IP & 0.311178 & HP-IP & 19.303 \\
LPA & 1.717340 & IP-LPA & 34.929 \\
LPB & 1.768430 & LPA-LPB & 52.038 \\
GEN & 1.736990 & LPB-GEN & 70.858 \\
EXC & 0.068433 & GEN-EXC & 2.822 \\
\hline
\end{tabular}

The natural frequencies of the torsional vibration of the turbogenerator shaft are 15.78, 20.22, $25.56,32.31$, and $47.46 \mathrm{~Hz}$, respectively.

The following parameters are given in p.u., unless stated otherwise.

B. Turbine prime mover parameters:

$F H P=0.3, F I P=0.26, F L P=0.44, T_{\text {css }}=0.3, T_{\text {rhs }}=7.0, T_{\text {cos }}=0.2$.

C. Transformer, series compensated transmission lines, and infinite power grid parameters

$P_{\text {base }}=892.4 M V A, X_{T}=0.14, R_{T}=0.01, X_{L}=0.56, R_{L}=0.02, X_{S}=0.06$.

D. Synchronous generator parameters:

$X_{d}=1.79, X_{q}=1.71, X_{a d}=1.66, X_{a q}=1.58, X_{f}=0.001, X_{D}=1.666, X_{g}=1.825, X_{Q}=1.695$, $R_{a}=0.0015, R_{f}=0.001, R_{D}=0.0037, R_{g}=0.0182, R_{Q}=0.0053$.

E. Control system parameters of unit side:

$K_{G}=25, T_{S R}=0.2, T_{S M}=0.3, K_{A}=50, T_{E}=0.002, T_{A}=0.1$.

F. System initial operating condition parameters:

$P_{\mathrm{e}}=0.9, V_{t}=1.05, \cos \varphi=0.9$ (lag).

\subsection{Eigenvalue Calculation Results}

Based on the above system parameters and initial state variables, the eigenvalue analysis method can be used to calculate all 27 eigenvalues of the system, which consists of nine pairs of conjugate complex numbers and nine real numbers. In this model, eigenvalues 1 to 12 (six pairs of conjugate 
complex numbers) represent the torsional dynamics of the turbogenerator shaft system. Eigenvalues 1 and 2 correspond to the low frequency oscillation mode (TM0). Eigenvalues 3 to 12 correspond to the torsional modes (TM1 to TM5). Eigenvalues 17 to 18 and 19 to 20 represent the supersynchronous and subsynchronous electric network modes, respectively.

The series capacitor compensation level of transmission lines is the dominant factor influencing the electrical damping of subsynchronous oscillations. The definition of compensation level $k_{C}$ for series capacitors given by

$$
k_{C}=\frac{X_{C}}{X_{L}}
$$

where $X_{C}$ and $X_{L}$ are the transmission lines capacitive reactance and inductive reactance, respectively.

When $k_{C}$ is 0.7 , the calculation results are shown in Table 3 (only 12 eigenvalues representing the low frequency and torsional modes of shaft are written in the table). As can be seen from Table 3 , the real parts of TM1 and TM2 are greater than zero, which means TM1 and TM2 are unstable, and their corresponding frequencies are $15.96 \mathrm{~Hz}$ and $20.38 \mathrm{~Hz}$, respectively. The system is in multimode subsynchronous resonance state. The real part of TM2 is the largest, indicating that the main oscillation frequency of the system is $20.38 \mathrm{~Hz}$.

Table 3. Eigenvalues calculation result $\left(k_{C}\right.$ is 0.7$)$.

\begin{tabular}{ccccc}
\hline Eigenvalue & Torsional Mode & Real Part (1/s) & Imaginary Part (rad/s) & Frequency (Hz) \\
\hline 1,2 & TM0 & -0.5073 & \pm 11.66 & 1.86 \\
3,4 & TM1 & 0.0931 & \pm 100.28 & 15.96 \\
5,6 & TM2 & 0.2034 & \pm 128.08 & 20.38 \\
7,8 & TM3 & -0.1352 & \pm 160.34 & 25.52 \\
9,10 & TM4 & -0.0404 & \pm 202.78 & 32.27 \\
11,12 & TM5 & -0.1818 & \pm 298.17 & 47.46 \\
\hline
\end{tabular}

To verify the validity of the eigenvalue analysis method, PSCAD time domain simulation method was used to analyze the above examples. For the case of $k_{C}$ is 0.7 , a disturbance was set on the electrical side. A three-phase short-circuit fault happened at $1.5 \mathrm{~s}$ and was released after $75 \mathrm{~ms}$. The simulation results are shown in Figure 3.

From Figure 3a, it can be noticed that when a three-phase short-circuit fault occurs, the electromagnetic torque of the generator produces a relatively large fluctuation and the amplitude continuously increases with time. From Figure $3 b$, it can be seen that the fluctuating electromagnetic torque excites the torsional vibration of the shaft, which is manifested by the amplitude oscillation of the shaft torque and divergence with time. It indicates that the system is no longer stable and develops subsynchronous oscillations after a three-phase short-circuit fault occurs. The torque between LP-A and B was analyzed by the fast Fourier transform (FFT). The results are shown in Figure 3c. It can be seen that the torsional vibration frequencies are mainly $15.96 \mathrm{~Hz}$ and $20.38 \mathrm{~Hz}$, and the amplitude of $20.38 \mathrm{~Hz}$ is the largest, which corresponds to TM1 and TM2. The result is in accordance with that of the eigenvalue analysis method. 


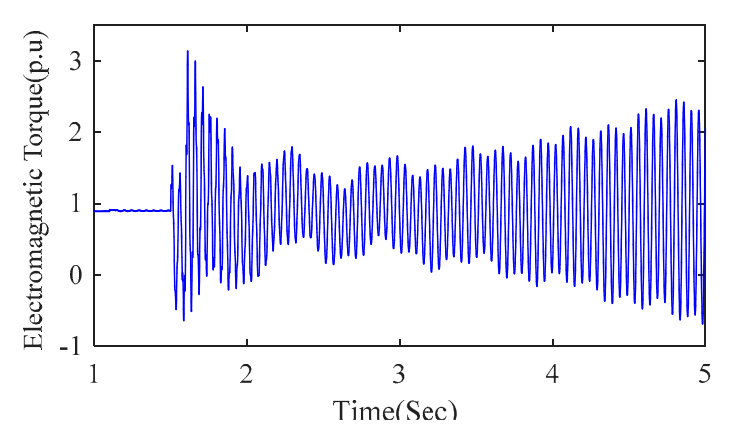

(a)

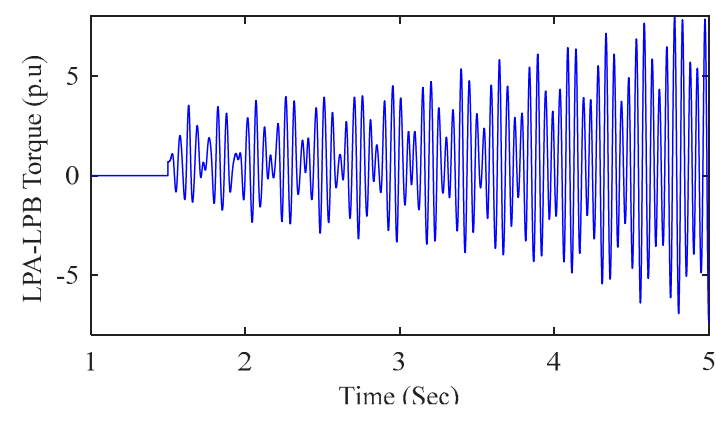

(b)

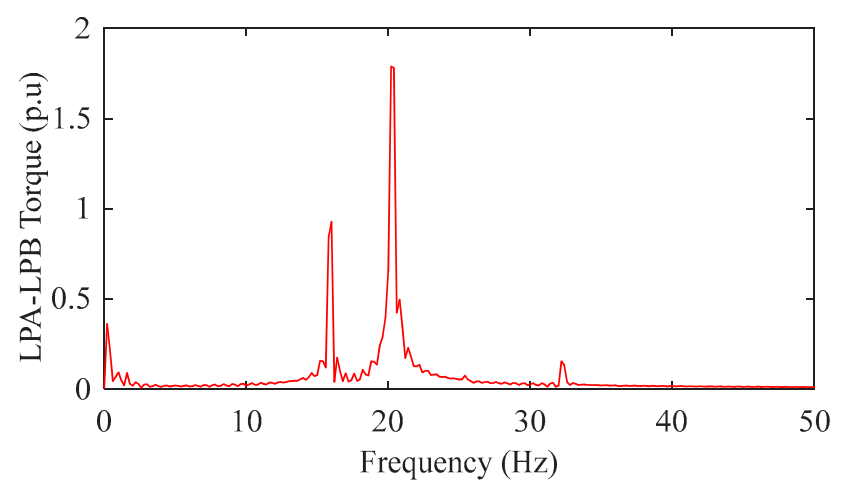

(c)

Figure 3. System response in three phase short circuit fault. (a) Generator electromagnetic torque. (b) LPA-LPB torque. (c) Fast Fourier transform (FFT) of the LPA-LPB torque.

\section{Analysis of Influence Factors of SSR}

\subsection{Influence of Compensation Level}

The eigenvalue analysis method was used to calculate the system eigenvalues with different compensation levels $k_{C}$, and the results are shown in Table 4 and Figure 4.

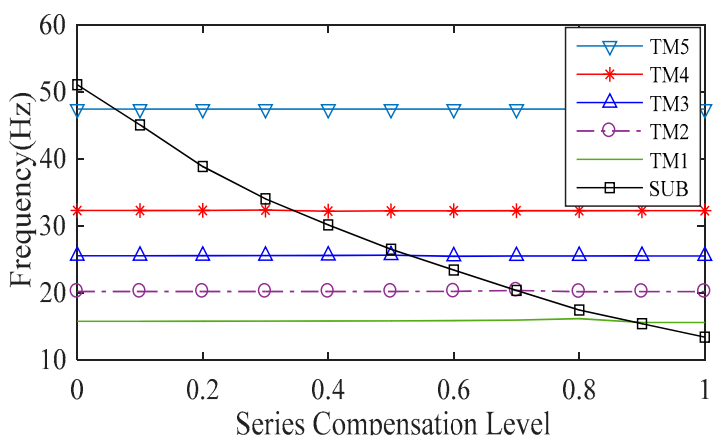

(a)

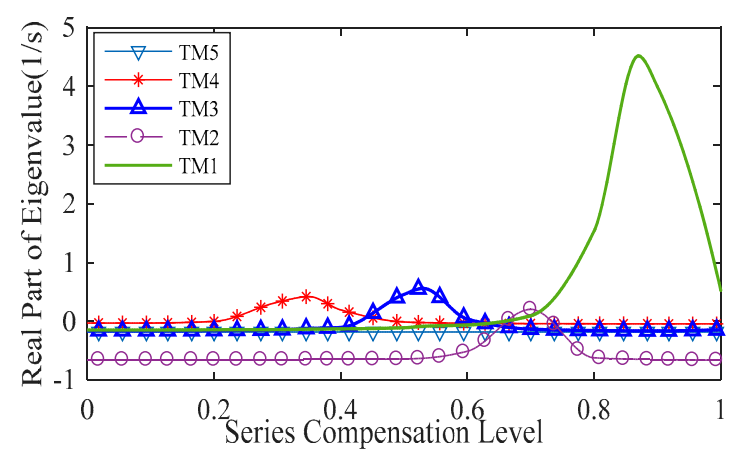

(b)

Figure 4. Influence of series compensation level on SSR characteristics. (a) The imaginary parts of eigenvalues. (b) The real parts of eigenvalues. 
Table 4. System eigenvalues with different compensation levels $K_{C}$.

\begin{tabular}{ccccccc}
\hline Eigenvalue & Torsional Mode & $\boldsymbol{K}_{\boldsymbol{C}}=\mathbf{0 . 1}$ & $\boldsymbol{K}_{\boldsymbol{C}}=\mathbf{0 . 3}$ & $\boldsymbol{K}_{\boldsymbol{C}}=\mathbf{0 . 5}$ & $\boldsymbol{K}_{\boldsymbol{C}}=\mathbf{0 . 7}$ & $\boldsymbol{K}_{\boldsymbol{C}}=\mathbf{0 . 9}$ \\
\hline 1,2 & TM0 & $0.0693 \pm \mathrm{j} 8.46$ & $-0.0748 \pm \mathrm{j} 9.31$ & $-0.2597 \pm \mathrm{j} 10.35$ & $-0.5073 \pm \mathrm{j} 11.66$ & $-0.8623 \pm \mathrm{j} 13.41$ \\
3,4 & TM1 & $-0.1487 \pm \mathrm{j} 99.13$ & $-0.1422 \pm \mathrm{j} 99.27$ & $-0.1106 \pm \mathrm{j} 99.52$ & $0.0931 \pm \mathrm{j} 100.28$ & $3.9947 \pm \mathrm{j} 98.05$ \\
5,6 & TM2 & $-0.6549 \pm \mathrm{j} 127.02$ & $-0.6525 \pm \mathrm{j} 127.03$ & $-0.6423 \pm \mathrm{j} 127.07$ & $0.2034 \pm \mathrm{j} 128.08$ & $-0.6522 \pm \mathrm{j} 126.92$ \\
7,8 & TM3 & $-0.1627 \pm \mathrm{j} 160.62$ & $-0.1479 \pm \mathrm{j} 160.69$ & $0.4564 \pm \mathrm{j} 161.08$ & $-0.1351 \pm \mathrm{j} 160.34$ & $-0.1644 \pm \mathrm{j} 160.46$ \\
9,10 & TM4 & $-0.0301 \pm \mathrm{j} 203.01$ & $0.3233 \pm \mathrm{j} 203.43$ & $-0.0125 \pm \mathrm{j} 202.68$ & $-0.0404 \pm \mathrm{j} 202.78$ & $-0.0449 \pm \mathrm{j} 202.84$ \\
11,12 & TM5 & $-0.1819 \pm \mathrm{j} 298.17$ & $-0.1819 \pm \mathrm{j} 298.17$ & $-0.1819 \pm \mathrm{j} 298.17$ & $-0.1819 \pm \mathrm{j} 298.17$ & $-0.1819 \pm \mathrm{j} 298.17$ \\
13,14 & SUB & $-7.1191 \pm \mathrm{j} 283.35$ & $-7.1702 \pm \mathrm{j} 213.94$ & $-7.0882 \pm \mathrm{j} 166.68$ & $-6.5888 \pm \mathrm{j} 128.06$ & $-9.1422 \pm \mathrm{j} 96.86$ \\
15,16 & SUPER & $-7.5026 \pm \mathrm{j} 470.47$ & $-7.5806 \pm \mathrm{j} 539.24$ & $-7.6238 \pm \mathrm{j} 586.55$ & $-7.6543 \pm \mathrm{j} 624.99$ & $-7.6778 \pm \mathrm{j} 658.22$ \\
17,18 & & $-4.6872 \pm \mathrm{j} 0.6335$ & $-4.7382 \pm \mathrm{j} 0.3856$ & $-4.8737 \pm \mathrm{j} 0.2566$ & $-4.9573 \pm \mathrm{j} 0.1521$ & $-3.4114 \pm \mathrm{j} 0.5459$ \\
19 & & -1.8966 & -1.8446 & -1.7833 & -1.6996 & -1.5619 \\
20 & & -24.7628 & -24.7842 & -24.8113 & -24.8466 & -24.8939 \\
21 & & -30.8793 & -31.5042 & -32.3627 & -33.6064 & -35.5306 \\
22 & & -8.5726 & -8.3123 & -7.9786 & -7.5383 & -6.9382 \\
23 & & -101.8931 & -101.7953 & -101.648 & -101.4514 & -101.1882 \\
24 & & -499.9582 & -499.9821 & -499.9783 & -499.9742 & -499.9713 \\
25 & & -2.9453 & -3.1215 & -3.4887 & -3.4828 \\
26 & & -0.1417 & -0.1417 & -0.1418 & -0.1419 & -3.9244 \\
27 & & -4.6223 & -4.3293 & -3.6731 & -0.1419 \\
sum & & -696.0893 & -696.1123 & -696.1281 & -696.0932 \\
\hline
\end{tabular}

Table 4 shows the system eigenvalues when $k_{C}$ is $0.1,0.3,0.5,0.7$ and 0.9 , respectively. Figure $4 \mathrm{a}$ describes the variation of the torsional mode frequency and the electrical resonance complementary frequency with $k_{C}$. In the figure, $f_{S U B}$ corresponds to the electrical resonance complementary frequency, and its calculation formula given by [11]

$$
f_{\text {SUB }}=f_{0}\left(1-\sqrt{\frac{X_{C}}{X^{\prime \prime}+X_{T}+X_{L}}}\right)
$$

where $f_{0}$ is the synchronous frequency, $X_{T}$ is the leakage reactance of the transformer, $X^{\prime \prime}$ is the subtransient reactance of the generator, and $X_{C}$ and $X_{L}$ are the transmission line inductive and capacitive reactance, respectively. Figure $4 \mathrm{~b}$ depicts the variation of the real part of eigenvalue with $k_{C}$.

It can be seen from Table 4 that eigenvalues are different in the case of different $k_{C}$, but all the sums of the eigenvalues are constant and the absolute value is about 696.1, which indicates that the total damping of the system is conserved, that is, the changes of $k_{C}$ do not affect the total system damping. Changing $k_{C}$ will cause various part damping of the system to be transferred and distributed between different locations, but the total system damping remains unchanged. When $k_{C}$ is small, the real part of TM0 is positive, the corresponding damping is negative, and the system is prone to low-frequency oscillations. This is because the total reactance of transmission lines is larger when $k_{C}$ is low. The electric distance of transmission lines is longer, and low frequency oscillations can occur if a power system stabilizer is not installed in the system. With the increase of $k_{C}$, it is equivalent to shortening the electrical distance of the transmission lines and enhancing the connection between generators and the system, and the risk of low frequency oscillations is reduced. When $k_{C}$ is greater than 0.3 , the real part of the TM0 eigenvalue is negative, which indicates that low-frequency oscillations do not occur in the system.

From Table 4 , it can be seen that when $k_{C}$ is equal to 0.3 , the real part of TM4 is positive (0.3233), and the oscillation frequency is $32.27 \mathrm{~Hz}$; when $k_{C}$ is equal to 0.5 , the real part of TM3 is positive (0.4564), and the oscillation frequency is $25.56 \mathrm{~Hz}$; When $k_{C}$ is equal to 0.7 , the real parts of TM2 and TM1 are positive, and the oscillation frequencies are $20.22 \mathrm{~Hz}$ and $15.78 \mathrm{~Hz}$, respectively; when $k_{C}$ is equal to 0.9 , the real part of TM1 is positive (3.9947) and the oscillation frequency is $15.78 \mathrm{~Hz}$. This shows that when $k_{C}$ is greater than 0.3 , at least one eigenvalue of the system is positive, that is, there is always a negative damping of a torsional mode. Then, the system is likely to cause SSR. With the increase of $k_{C}$, the dominant oscillation frequency decreases.

From Figure $4 a$, it can be noticed that the imaginary parts of TM1 to TM5's eigenvalues change very little in the process of $k_{C}$ being changed from zero to one, that is, the torsional mode frequencies $\left(f_{T M x}\right)$ of the turbogenerator shaft remain basically unchanged. The reason is that the torsional natural 
frequencies are mainly determined by the turbogenerator shaft structure parameters, and the torsional natural frequencies will be determined after the turbogenerator is manufactured. The electrical resonance complementary frequency $\left(f_{S U B}\right)$ decreases with the increase of $k_{C}$. The changed amplitude is relatively large, and it will generate intersections with different torsional resonant frequencies. At these intersections, the torsional frequency and the electrical resonant frequency are complementary.

From Figure $4 \mathrm{~b}$, it can be noticed that the damping of TM1 to TM5 vary greatly. The closer $f_{T M}$ is to $f_{S U B}$, the larger the real part of the eigenvalue is. When $f_{T M}$ is equal to $f_{S U B}$, the real part of the eigenvalue reaches the maximum, which means that the risk of torsional vibration is maximum. The real part of TM5 eigenvalue $(47.46 \mathrm{~Hz})$ is negative in the process of $k_{C}$ being changed from 0 to 1 , and it will not cause instability. The other four SSR modes will cause instability, and $k_{C}$ of instability areas are as follows: For TM $4(32.31 \mathrm{~Hz}), k_{C}$ of instability areas is between 0.21 and 0.447 , and instability is the most serious when $k_{C}$ is 0.35 . For TM3 $(25.56 \mathrm{~Hz}), k_{C}$ of instability areas is between 0.43 and 0.64 , and instability is the most serious when $k_{C}$ is 0.53 . For TM2 $(20.22 \mathrm{~Hz}), k_{C}$ of instability areas is between 0.67 and 0.72 , and instability is the most serious when $k_{C}$ is 0.71 . For TM1 $(15.78 \mathrm{~Hz}), k_{C}$ of instability areas is between 0.62 and 1.0, and instability is the most serious when $k_{C}$ is 0.86 . Due to the overlapping of instability intervals, the turbogenerator may generate multimode SSR instability problems, for example, when $k_{C}$ is 0.7 , both TM2 and TM1 are in a instability state.

\subsection{Influence of Synchronous Generator Parameters}

The parameters of synchronous generators include reactance and resistance parameters. The reactance parameters mainly include stator leakage reactance $X_{l}, d$-axis synchronous reactance $X_{d}, q$-axis synchronous reactance $X_{\mathrm{q}}, d$-axis armature reaction reactance $X_{a d}, q$-axis armature reaction reactance $X_{a q}$, excitation winding reactance $X_{f}, d$-axis damper winding $D$ reactance $X_{D}, q$-axis damper winding $g$ reactance $X_{g}$, and $q$-axis damper winding $Q$ reactance $X_{Q}$. The resistance parameters mainly include armature winding resistance $R_{a}$, excitation winding resistance $R_{f}, d$-axis damper winding $D$ resistance $R_{D}, q$-axis damper winding $g$ resistance $R_{g}$, and $q$-axis damper winding $Q$ resistance $R_{Q}$. Among them, the study of parameters $X_{d}$ and $X_{\mathrm{q}}$ for SSR can be converted into the analysis of parameter $X_{l}$. Taking $k_{C}$ as 0.5 , the generator parameters vary from $50 \%$ to $150 \%$ of nominal value. The eigenvalue analysis method was used to calculate the eigenvalues of five torsional modes, and the results are shown in Figure 5.

It can be seen from Figure $5 \mathrm{a}$ that the frequency of each torsional mode remains basically unchanged when $X_{l}$ increases, and the other electrical parameters have the same effect on the torsional frequency, which means that the torsional frequency of the turbogenerator shaft is basically irrelevant to the reactance parameters. It can be seen from Figure $5 b$ that the real part of TM3 eigenvalue decreases slightly when $X_{l}$ increases, and the corresponding negative damping decreases, which is beneficial to the stability of TM3.

The real part of TM4 eigenvalue increases, which means positive damping decreases and negative damping increases. When $X_{l}$ is greater than 1.2, the real part of TM4 eigenvalue changes from negative to positive and TM4 oscillation is excited. The system presents a multimode resonance state in which TM3 and TM4 coexist. The reason is that the $f_{S U B}$ increases continuously when $X_{l}$ increases, which transitions from near $f_{T M 3}$ to near $f_{T M 4}$.

The influence of reactance parameters $X_{f}, X_{D}, X_{g}$ and $X_{Q}$ on the eigenvalues is similar, so Figure $5 c$ shows only the influence of $X_{f}$. It can be seen from the Figure $5 c$, the influence of reactance parameters $X_{f}$ on the eigenvalues is similar to $X_{l}$, but their influence on the real parts of the eigenvalues is less than $X_{l}$.

Figure $5 \mathrm{~d}$ describes the effect of the generator resistance parameter $R_{a}$ on the eigenvalues, and it can be seen that $R_{a}$ has little effect on the real parts of the eigenvalues, because $R_{a}$ is much smaller than the line resistance and has very little effect on the torsional modes. The effect of other resistance parameters is similar to that of $R_{a}$. 
In conclusion, neither the generator reactance parameters nor the resistance parameters will affect the torsional frequencies of the shaft system. When the reactance parameters of the generator change, which makes $f_{S U B}$ and $f_{T M x}$ close to each other, the SSR instability may occur. However, it will increase the damping of the adjacent torsional modes, which is beneficial to its stability. The resistance parameters of synchronous generator have little effect on each torsional mode and it can be ignored.

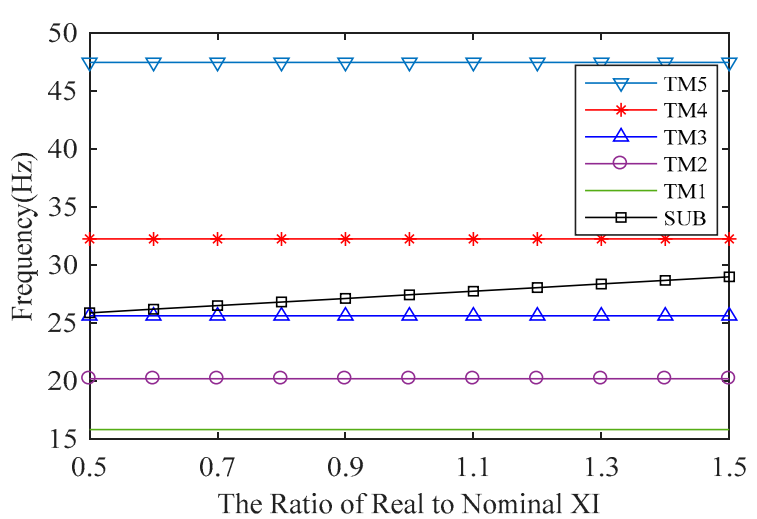

(a)

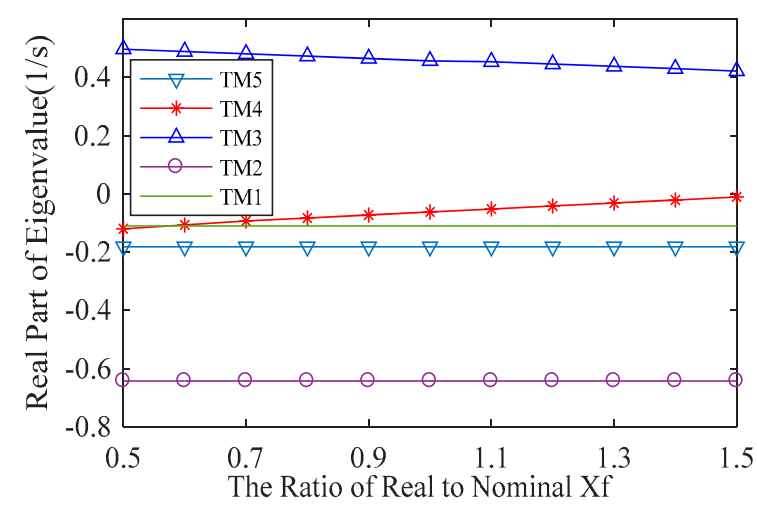

(c)

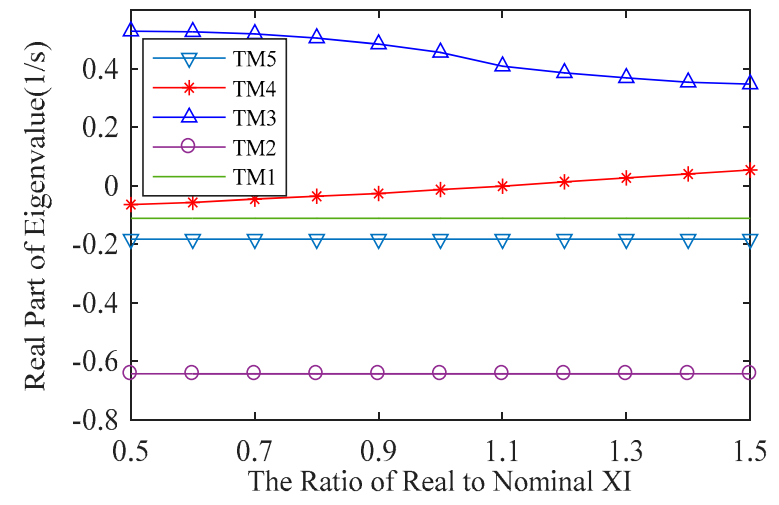

(b)

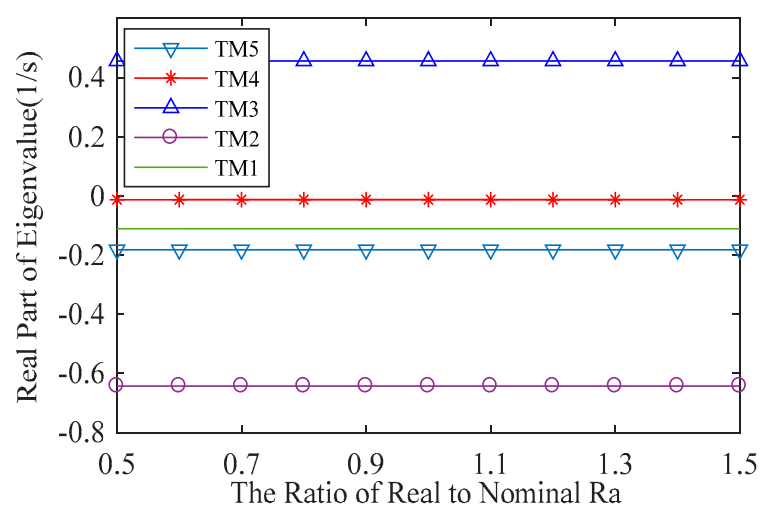

(d)

Figure 5. Influence of synchronous generator parameters on SSR characteristics. The imaginary parts of eigenvalues affected by (a) $X_{l}$, the real parts of eigenvalues affected by (b) $X_{l}$, (c) $X_{f}$, (d) $R_{a}$.

\subsection{Influence of Speed Governing System Parameters}

\subsubsection{With or Without Speed Governing System}

The system models with and without a speed governing system were established, respectively. The eigenvalue analysis method was used to calculate the eigenvalues when $k_{C}$ is 0.5 . The results are shown in Table 5. It can be seen from the Table that when there is a speed governing system, the real part of each eigenvalue is slightly increased, which indicates that the existence of the speed governing system reduces the system damping and results in poor system stability. The speed governing system has a significant impact on the TM0, reducing the TM0 damping and frequency. 
Table 5. Eigenvalues with and without speed governing system.

\begin{tabular}{ccccc}
\hline \multirow{2}{*}{ Torsional Mode } & \multicolumn{2}{c}{ With Speed Governing System } & \multicolumn{2}{c}{ Without Speed Governing System } \\
\cline { 2 - 5 } & Real Part (1/s) & Imaginary Part (rad/s) & Real Part (1/s) & Imaginary Part (rad/s) \\
\hline TM0 & -0.2597 & 10.35 & -0.3099 & 10.56 \\
TM1 & -0.1818 & 298.17 & -0.1828 & 298.17 \\
TM2 & -0.0125 & 202.68 & -0.0153 & 202.68 \\
TM3 & 0.4564 & 161.08 & 0.4453 & 161.08 \\
TM4 & -0.6423 & 127.07 & -0.6505 & 127.07 \\
TM5 & -0.1106 & 99.52 & -0.1224 & 99.52 \\
\hline
\end{tabular}

\subsubsection{Influence of Speed Governing System Parameters}

The parameters of the speed governing system mainly include $K_{G}, T_{S R}$ and $T_{S M}$. When $k_{C}$ is 0.5 , $K_{G}$ changes from 20 to $30, T_{S R}$ and $T_{S M}$ changes from $50 \%$ to $150 \%$ of nominal value. The eigenvalue analysis method was used to calculate system eigenvalues. The results are shown in Figure 6. It can be noticed that $K_{G}, T_{S R}$ and $T_{S M}$ have little effect on the imaginary part of each eigenvalue, and the real part increases slightly. With the increase of $K_{G}$ and the decrease of $T_{S R}$ and $T_{S M}$, TMx damping is slightly reduced, which increases the risk of SSR in the system. The reason is that the smaller $T_{S R}$ and $T_{S M}$ are, the shorter the reaction time of the speed governing system is and the faster the response of the shaft system to the electrical resonance is, which is beneficial to the transmission of SSR energy.

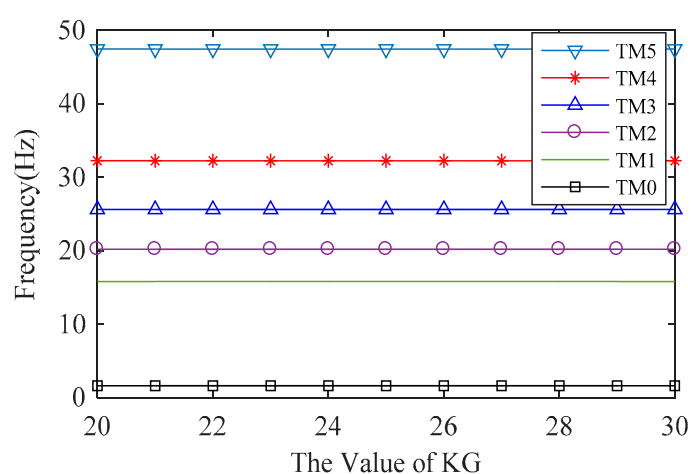

(a)

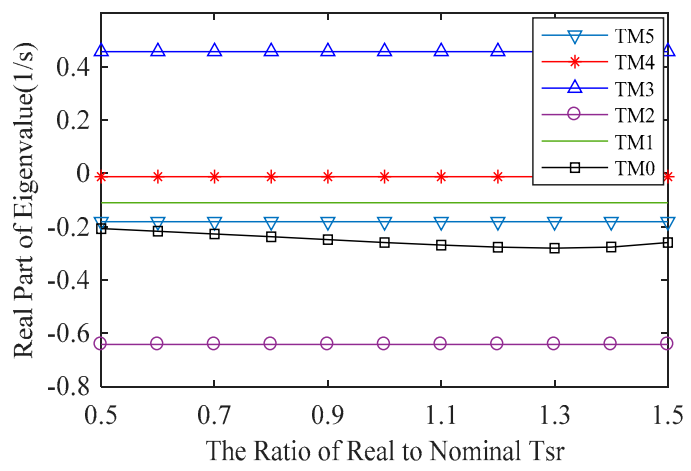

(c)

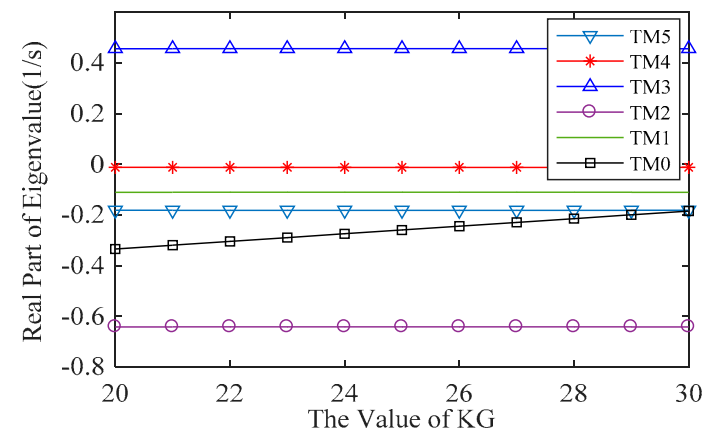

(b)

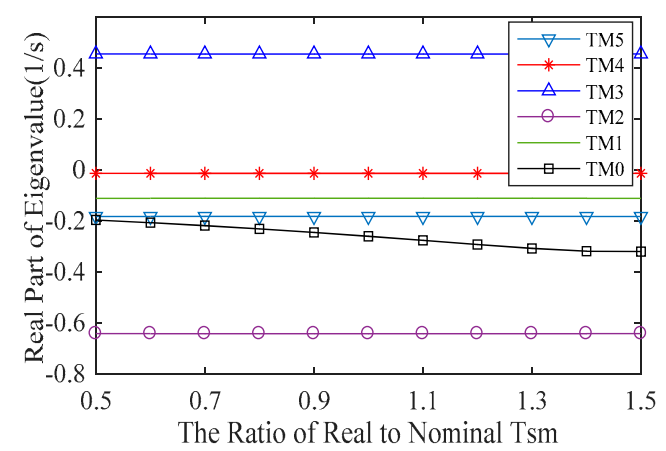

(d)

Figure 6. Influence of speed governing system parameters on SSR characteristics. The imaginary parts of eigenvalues affected by (a) $K_{G}$, the real parts of eigenvalues affected by (b) $K_{G}$, (c) $T_{S R}$, (d) $T_{S M}$.

$K_{G}, T_{S R}$ and $T_{S M}$ have a large influence on TM0. With the increase of $K_{G}$ and the decrease of $T_{S R}$ and $T_{S M}$, the real part of TM0 eigenvalue increases significantly, that is, TM0 damping decreases more, which means that the risk of low frequency oscillations in the system is increased. 


\subsection{Influence of Excitation System Parameters}

In the excitation regulator, the magnification of the excitation regulator $K_{A}$ is an important parameter. Taking $k_{C}$ as 0.5 and changing $K_{A}$ from 25 to 75 , the eigenvalue analysis method was used to calculate eigenvalues of the system, and the result is shown in Figure 7.

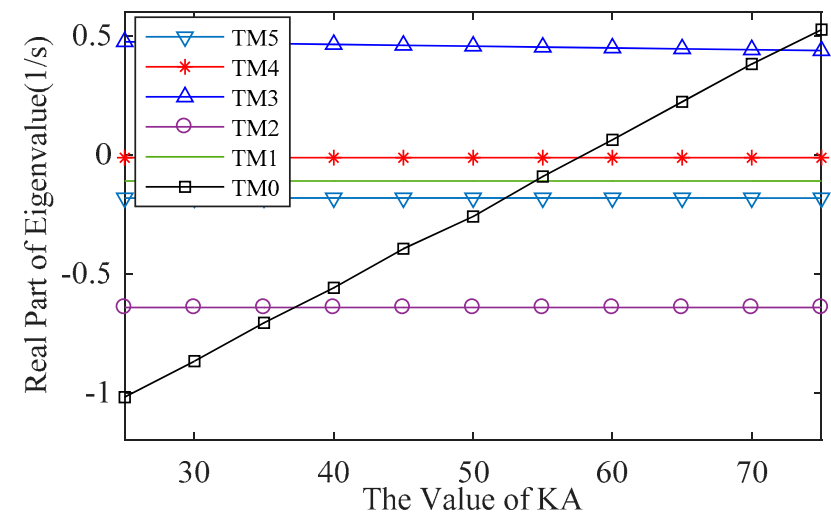

Figure 7. Influence of excitation system parameters on SSR characteristics.

As can be seen from Figure 7, with the increase of $K_{A}$, the real part of TM3 eigenvalue decreases. That is to say, the negative damping of TM3 in the divergence mode decreases, which means that increasing $K_{A}$ can reduce the instability of SSR and reduce the risk of SSR. With the increase of $K_{A}$, the real part of TM0 eigenvalue increases significantly, that is, the damping value of TM0 is obviously reduced. When $K_{A}$ is greater than 58 , the real part of TM0 eigenvalue even changes from negative to positive, and the system has the risk of generating divergent low-frequency oscillations.

\section{Conclusions}

The purpose of this paper was to study the SSR characteristics and influence factors of the series compensation transmission system. By using the eigenvalue analysis method and time domain simulation method, the eigenvalues of a series compensated transmission system were calculated, and the following conclusions can be drawn:

- The series compensation level $k_{C}$ has the greatest influence on the torsional mode damping of the system. With the increase of $k_{C}$, one or more torsional mode damping will appear negative, indicating that the system may have a single mode or multimode SSR, and the greater $k_{C}$ is, the lower the torsional mode frequency is, but the change of $k_{C}$ does not affect total damping of the system, that is, the total damping of the system is conserved.

- The parameters of the synchronous generator will not affect torsional mode frequencies of the turbogenerator shaft, and the reactance parameters of generators have a certain influence on torsional mode damping of the system. The variation of the reactance parameters may lead to instability of the torsional mode when $f_{S U B}$ and $f_{T M x}$ become close. However, the damping of adjacent torsional modes will be increased, which is beneficial to its stability. In addition, the resistance parameters of synchronous generator have little effect on the damping of each torsional mode, which can be ignored.

- Speed governing system parameters $K_{G}, T_{S R}$ and $T_{S M}$ have a great influence on TM0 damping, and slightly affect other torsional modes damping. Excitation system parameter $K_{A}$ significantly affects TM0 damping, and has little effect on other torsional modes damping.

Author Contributions: All the authors were involved in the data collection, model constructing, software simulation, results analysis and discussion, and manuscript preparation. All authors have approved the submitted manuscript. 
Funding: This work is supported by the Open Research Fund of Key Laboratory of Space Utilization, Chinese Academy of Sciences (No. LSU-2016-04-02) and the Natural Science Foundation of Beijing, China (Grant No. 3132015).

Conflicts of Interest: The authors declare no conflict of interest.

\section{References}

1. Bizzarri, F.; Brambilla, A.; Milano, F. Simplified model to study the induction generator effect of the subsynchronous resonance phenomenon. IEEE Trans. Energy Conver. 2018, 33, 889-892. [CrossRef]

2. Kumar, R.; Harada, A.; Merkle, M.; Miri, A.M. Investigation of the influence of series compensation in AC transmission systems on the bus connected parallel generating units with respect to subsynchronous resonance (SSR). In Proceedings of the 2003 IEEE Bologna Power Tech Conference Proceedings, Bologna, Italy, 23-26 June 2003; pp. 1083-1088.

3. Li, J.; Zhang, P.X. Impact of increased wind power generation on subsynchronous resonance of turbine-generator units. J. Mod. Power Syst. Clean Energy. 2016, 4, 219-228. [CrossRef]

4. Virulkar, V.B.; Gotmare, G.V. Sub-synchronous resonance in series compensated wind farm: A review. Renew. Sustain. Energy Rev. 2016, 55, 1010-1029. [CrossRef]

5. Xie, X.R.; Zhang, X.; Liu, H.K.; Liu, H.; Li, Y.H.; Zhang, C.Y. Characteristic analysis of subsynchronous resonance in practical wind farms connected to series-compensated transmissions. IEEE Trans. Energy Conver. 2017, 32, 1117-1126. [CrossRef]

6. Zhu, B.C.; Hu, M.; Wu, Z. Parameters impact on the performance of a double-fed induction generator-based wind turbine for subsynchronous resonance control. IET Renew. Power Gener. 2012, 6, 92-98. [CrossRef]

7. Chen, W.H.; Xie, X.R.; Wang, D.H. Probabilistic stability analysis of subsynchronous resonance for series compensated DFIG-based wind farms. IEEE Trans. Sustain. Energ. 2018, 9, 400-409. [CrossRef]

8. Park, J.K.; Kim, C.H.; Cho, G.J.; Sohn, S.H.; Chung, S.J. A Novel Reclosing Algorithm Considering Turbine-Generator Shaft Torque. IEEE Trans. Power Deliv. 2017, 32, 703-712. [CrossRef]

9. Dong, D.Y.; Chen, W.H.; Zhang, J.; Tan, L.N. Subsynchronous resonance modeling method for real systems. Power Syst. Tech. 2015, 39, 1059-1066.

10. Johansson, N.; Ängquist, L.; Nee, H.P. A comparison of different frequency scanning methods for study of subsynchronous resonance. IEEE Trans. Power Syst. 2011, 26, 356-363. [CrossRef]

11. Li, H.F.; Liu, C.R.; Han, M.X. Analysis on subsynchronous resonance caused by series compensation in AC system based on frequency-scanning and eigenvalue. Power Syst. Tech. 2012, 36, 107-112.

12. IEEE Subsynchronous Resonance Task Force. First benchmark model for computer simulation of subsynchronous resonance. IEEE Trans. Power Appar. Syst. 1977, 96, 1565-1572. [CrossRef]

13. Yu, Y.X.; Li, J. The power system subsynchronous oscillations were studies based on eigenvalue analysis. Electr. Eng. 2017, 18, 44-48.

(C) 2019 by the authors. Licensee MDPI, Basel, Switzerland. This article is an open access article distributed under the terms and conditions of the Creative Commons Attribution (CC BY) license (http://creativecommons.org/licenses/by/4.0/). 